\title{
Texture Classification based on Local Features Using Dual Neighborhood Approach
}

\author{
M. Srinivasa Rao \\ Associate Professor, Dept. of C.S.E, Sri Vasavi Institute of Engineering \& Technology, pedana, Andhrapradesh, India \\ Email: srinu.mekala@gmail.com \\ V.Vijaya Kumar \\ Professor, Anurag Group of Institutions (Autonomous), Hyderabad,Telanagana, India \\ Email: vakula_vijay@yahoo.com \\ MHM KrishnaPrasad \\ Professor of the Department of Computer Science and Engineering, University College of Engineering, Kakinada \\ (Autonomous), JNTUK, Andhra Pradesh, India \\ Email: krishnaprasad.mhm@gmail.com
}

Received: 11 March 2017; Accepted: 05 July 2017; Published: 08 September 2017

\begin{abstract}
Texture classification and analysis are the most significant research topics in computer vision. Local binary pattern (LBP) derives distinctive features of textures. The robustness of LBP against gray-scale and monotonic variations and computational advantage have made it popular in various texture analysis applications. The histogram techniques based on LBP is complex task. Later uniform local binary pattern's (ULBP) are derived on LBP based on bit wise transitions. The ULBP's are rotationally invariant. The ULBP approach treated all non-uniform local binary pattern's (NULBP) into one miscellaneous label. This paper presents a new texture classification method incorporating the properties of ULBP and grey-level co-occurrence matrix (GLCM). This paper derives ternary patterns on the ULBP and divides the $3 \times 3$ neighborhood in to dual neighborhood. The ternary pattern mitigates the noise problems particularly near uniform regions. The dual neighborhood reduces the range of texture unit from 0 to 6561 to 0 to 80 . The GLCM features extracted from ULBP-dual texture matrix (ULBP-DTM) provide complete texture information about the image and reduce the texture unit range. Various machine learning classifiers are used for classification purpose. The performance of the proposed method is tested on Brodtaz, Outex and UIUC's textures and compared with GLCM, texture spectrum (TS) and cross-diagonal texture matrix (CDTM) approaches.
\end{abstract}

Index Terms - Local binary pattern (LBP), Uniform LBP (ULBP-DTM), ternary patter, dual neighborhood, texture spectrum (TS).

\section{INTRODUCTION}

Texture is one of the very fundamental and important appearances of the object surface. Texture is widely used in object surface recognition and description. Texture classification plays an important role in texture analysis. Classification and analysis of textures has wide range of applications, for example, in industrial inspections, medical image processing, face recognition, problems related to human faces like face, facial expression, age classification issues, remote sensing, etc. The texture classification method initially compares the features of a unknown test sample, with the training sample features with respect to spatial scale, orientation, grey scale properties etc... Then the unknown sample is given the class of training sample that is more identical or whose distance measure is least. Texture classification mainly dependent on the features extracted on the image or object surface. Numerous methods are derived in the past decades, to extract the features from local patterns in texture classification [1-5].

In the literature rotation invariant approaches has been developed. Circular auto regressive model are initially used to derive rotation-invariant texture classification [2]. Recently texton distribution methods are used for texture classification [3, 6, 7] and also for age classification [8].The revolutionary work of Ojala et al. "local binary pattern (LBP)" is extensively used for rotation invariant texture classification these days [9]. The LBP attracted increasing attention among the research scholars because of low computational complexity and invariance to monotonic illumination changes and rotation. The rotation invariant uniform LBP (LBPriu2), proposed by Ojala achieved a good rotational invariance performances. Later the original LBP operator [10] has been extended in several ways, such as neighborhoods with different sizes [9], multi-resolution [11], uniform patterns [12], prominent- LBP [31] etc. These developments on LBP mostly focus at capturing more discriminative and rotationally invariant information in local patterns and has a wide application, such as texture analysis and 
classification [13-16], face detection and recognition [17 - 25], image retrieval [26], etc.

The other variant of LBP called local ternary pattern (LTP) [27] quantized the difference between a pixel and its neighbors into three levels. Some variants of LBP, such as derivative-based LBP [28], dominant LBP [29] and center-symmetric LBP [30], prominent LBP [31], dual LBP [26] have been proposed recently to overcome the noise and other related problems of LBP. The PLBP [31] derived new transitions that contains set of ULBP's and NULBP's and achieved prominent results in face recognition. Another variant of LBP descriptors [32], combined LBP and co-occurrence features. In local quantized patterns (LQP) [33], a clustering method was adopted. The local color vector binary pattern (LCVBP) [34] is especially developed for color image applications. A texture classification method named local vector quantization pattern [LQVP] [35] developed recently aimed at quantizing the whole difference vector between the central pixel and its neighborhood pixels instead of each neighborhood pixels separately and it attained a good classification result on UIUC, Outex and Brodatz data bases.

Randen and Huso [36] after an extensive comparative study involving dozens of different spatial filtering methods concluded that the degree of computational complexity of most proposed texture measures in the literature is too high. To address this, the paper proposes a new feature description called ULBP-dual texture matrix (ULBP-DTM) for rotation and gray-scale invariant texture classification. At first, our approach quantizes the given texture image into ULBP coded image, this preserves the rotational invariant and fundamental units of texture image. The next step further quantizes the given ULBP texture image with ternary values and evaluates texture units by splitting the each 3 $\mathrm{x} 3$ neighborhood in to dual neighborhood (cross and diagonal neighborhoods) of 4 pixels each. The GLCM features are evaluated on ULBP-DTM by using machinelearning classifiers for texture classification.

The rest of the paper is organized as follows. Section 2 briefly introduces LBP and its limitations; section 3 presents the derivation of ULBP-DTM. In Section 4 we present experimental results of proposed method and discussion. Finally, conclusions are drawn in Section 5.

\section{LOCAL BINARY PATTERNS}

LBP is a simple and efficient operator to describe local image pattern and it is a robust, theoretically and computationally simple approach. It brings together the separate statistical and structural approaches to texture analysis and this has resulted, a door for the analysis of both stochastic micro textures and deterministic macro textures simultaneously. The LBP has shown outstanding performance in many comparative studies, in terms of both discrimination performance and computational complexity. That's why many researchers in the field of image and video processing are motivated to use LBP operator in their findings.
The derivation of the LBP follows that represented by Ojala et al. [9]. There is no universally accepted definition for texture. In general a texture $\mathrm{T}$ in a local neighborhood of a gray-scale image is defined as the joint distribution of the gray levels of $\mathrm{P}+1(\mathrm{P}>0)$ image pixels:

$$
\mathrm{T}=\mathrm{t}\left(\mathrm{P}_{\mathrm{c}}, \mathrm{P}_{0}, \ldots, \mathrm{P}_{\mathrm{n}}\right),
$$

Where $P_{c}$ represents to the gray value of the center pixel and $P_{n}(n=0, \ldots, n-1)$ represent to the gray values of $\mathrm{P}$ equally spaced neighboring pixels on a circle of radius $R(R>0)$. That is according to LBP a texture is defined not only by the grey level values of a pixel $P_{c}$ also by the surrounding or neighboring pixels of $P_{c}$. The neighboring pixel coordinates are given by $\left(\mathrm{x}_{\mathrm{c}}+\right.$ $\left.\mathrm{R}^{*} \cos (2 \pi \mathrm{p} / \mathrm{P}), \mathrm{yc}-\mathrm{R}^{*} \sin (2 \pi \mathrm{p} / \mathrm{P})\right)$, where $\left(\mathrm{x}_{\mathrm{c}}, \mathrm{y}_{\mathrm{c}}\right)$ are the coordinates of the center pixel. The values of neighbors that do not fall exactly on pixels are estimated by bilinear interpolation. Most of the textural information in an image can be obtained from local neighborhoods because correlation between pixels decreases with distance.

One can represent the local texture, based on the difference of grey level values between center pixel and neighboring pixels:

$$
\mathrm{T}=\mathrm{t}\left(\mathrm{P}_{\mathrm{c}}, \mathrm{P}_{0}-\mathrm{P}_{\mathrm{c}}, \ldots, \mathrm{P}_{\mathrm{n}}-\mathrm{P}_{\mathrm{c}}\right)
$$

The above representation of texture is not true due to the limited nature of the values in digital images, further this representation describes the overall luminance of an image, which is unrelated to local image texture and does not provide useful information. The LBP measures only the signs of the differences, to achieve invariance with respect to any monotonic transformation of the grey scales. The LBP operator assigns a binomial weight $2^{\mathrm{p}}$ to each sign $\mathrm{S}\left(\mathrm{P}_{\mathrm{n}}-\mathrm{P}_{\mathrm{c}}\right)$. This transforms the sign differences in a neighborhood into a unique LBP code. The code characterizes the local image texture around $\left(\mathrm{x}_{\mathrm{c}}, \mathrm{y}_{\mathrm{c}}\right)$. In practice, Eq. 4 represents the LBP code or local grayscale distribution which results in $2^{\mathrm{n}}$ distinct values. The process of formation of LBP code is shown in the Fig.1.

$$
\mathrm{T} \sim \mathrm{t}\left(\mathrm{S}\left(\mathrm{P}_{0}-\mathrm{P}_{\mathrm{c}}\right), \ldots, \mathrm{S}\left(\mathrm{P}_{\mathrm{n}-1}-\mathrm{P}_{\mathrm{c}}\right)\right)
$$

where

$$
\begin{aligned}
& S(x)=1 \text { if } x>=0 \text { else } 0 \\
& \operatorname{LBP}_{\mathrm{P}, \mathrm{R}}\left(\mathrm{x}_{\mathrm{c}}, \mathrm{y}_{\mathrm{c}}\right)=\sum_{i=0}^{n-1} S\left(P_{i}-P_{c}\right) * 2^{i} \\
& \begin{array}{|l|l|l|}
\hline Z_{0} & Z_{1} & Z_{2} \\
\hline Z_{3} & Z_{\mathrm{c}} & Z_{4} \\
\hline Z_{5} & Z_{6} & Z_{7} \\
\hline
\end{array} \\
& \begin{array}{|l|l|l|}
\hline \mathrm{y}_{0} & \mathrm{y}_{1} & \mathrm{y}_{2} \\
\hline \mathrm{y}_{7} & & \mathrm{y}_{3} \\
\mathrm{y}_{6} & \mathrm{y}_{5} & \mathrm{y}_{4} \\
\hline
\end{array} \quad \begin{array}{|l|l|l|l|}
\hline 2^{0} & 2^{1} & 2^{2} \\
\hline 2^{7} & & 2^{3} \\
\hline 2^{6} & 2^{5} & 2^{4} \\
\rightarrow
\end{array} \rightarrow \begin{array}{l}
\text { LBP } \\
\text { code }
\end{array}
\end{aligned}
$$

Fig.1. Representation of LBP. 


\begin{tabular}{|l|l|l|}
\hline 65 & 89 & 20 \\
\hline 12 & 40 & 16 \\
\hline 96 & 28 & 36 \\
\hline
\end{tabular}$\rightarrow$\begin{tabular}{|l|l|l|}
\hline 1 & 1 & 0 \\
\hline 0 & & 0 \\
\hline 1 & 0 & 0 \\
\hline
\end{tabular}$\times$\begin{tabular}{|l|l|l|}
\hline $2^{0}$ & $2^{1}$ & $2^{2}$ \\
\hline $2^{7}$ & & $2^{3}$ \\
\hline $2^{6}$ & $2^{5}$ & $2^{4}$ \\
\hline
\end{tabular}$\rightarrow 67$

Fig.2. The derivation of basic LBP code.

A $3 \times 3$ neighborhood with 8-neighboring pixels derives a LBP code that ranges from 0 to $2^{8}-1=0$ to 255 . The Fig. 2 illustrates the process of LBP code derivation. The centre pixel 40 is replaced by the obtained LBP value 67. In this way a new LBP image is constructed by replacing the centre value with the corresponding LBP code. There is no unique way to label the coefficients and ordering the 255 LBP codes on a $3 \times 3$ neighborhood. The eight elements may be ordered differently. If the eight elements are ordered clock wise around the central pixel, the first element may take eight possible positions from top left corner to the left middle. This will result labeling of LBP code in 8-different ways. This will result 8different LBP codes for the Fig.2 \{13, 26, 52, 104, 208, $161,67,134\}$.

\section{The DERIVATION OF ULBP-DTM}

The number of LBP codes will be increased drastically if one increases the number of neighboring pixels $(\mathrm{P})$ from $8\left(2^{8}\right)$ to $16\left(2^{16}\right)$ or $24\left(2^{24}\right)$ over a radius $\mathrm{R}$. To quantify the varying performance of individual pattern attributes to the spatial structure of patterns the uniformity measure ' $U$ ' on LBP is defined. The uniformity corresponds to the number of circular spatial transitions (bitwise 0/1 changes) in the LBP. A LBP is treated as Uniform LBP (ULBP) if it contains at most two transitions from 0 to 1 or 1 to 0 in a circular manner. The remaining patterns are treated as non-uniform LBP (NULBP). The Pattern with 00000000, 01000000 and 01010101, derives 0,2 and 8 transitions respectively. A LBP with $(\mathrm{P}, \mathrm{R})$ where $\mathrm{P}=8$ and $\mathrm{R}=1$ will have $\mathrm{P} *(\mathrm{P}-1)$ $+2=58$ ULBPs and 198 NULBP's. Some of the researchers [37, 38] considered only ULBP's because of the following reasons. a) The fundamental properties of texture image can be precisely represented by ULBP. b) 90 to $95 \%$ of the texture images hold only ULBP windows. c) Treating the 192 NULBP's as miscellaneous will reduce lot of dimensionality without losing the texture content. The present paper given unique code for each ULBP i.e. from 1 to 58 and the code 0 is assigned to all remaining 198 NULBP's. The quantization process of the basic $3 \times 3$ neighborhood in to ULBP coded image is shown in Fig.3. Here the central pixel 75 is replaced with zero (NULBP code) because the LBP window is a NULBP.
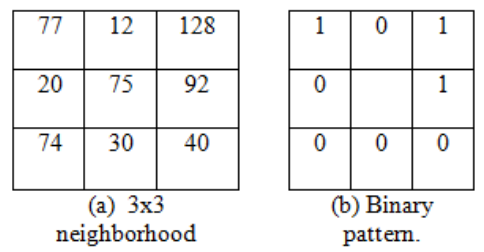

0

(c) ULBP quantization
The proposed ULBP-DTM initially quantizes the given grey level image in to ULBP window, by treating all noisy and non significant windows i.e. NULBPs in to miscellaneous. This quantization process represents the significant information of texture in the form of lines, curves, spots, blobs, edges etc... In the second step this paper further quantizes the ULBP coded image in to ternary representation by assigning one of three possible values (ternary patterns; 0,1 , and 2 ) to the neighboring pixels of ULBP windows i.e. using a threshold i.e. the grey level value of center pixel as specified in the equation_5.

$$
\begin{gathered}
T_{i}=\left\{\begin{array}{c}
0 \text { if } P_{i}<P_{c} \\
1 \text { if } P_{i}==P_{c} \text { for } i=0,1,2, \ldots 7 \\
2 \text { if } P_{i}>P_{c}
\end{array}\right. \\
\text { TUn }=\sum_{\mathrm{i}=0}^{7} \mathrm{~T}_{\mathrm{i}} * 3^{\mathrm{i}}, \mathrm{T}_{\mathrm{i}} \in\{0,1,2\}
\end{gathered}
$$

and

$$
\mathrm{TU}_{\mathrm{n}} \in\left\{0,1,2, \ldots 3^{8-1}\right\}
$$

By assigning ternary weights to the ULBP neighborhood it generates a unique code ranging from 0 to 6561 as given in equation 6 . The above ternary pattern approach on ULBP overcomes the sensitive to noise problem of LBP, which is particularly in the presence of smooth and weak illumination gradients in near uniform regions. That is ternary representation can better tolerate noise and it is significantly more distinctive. However one of the main disadvantages of this approach is it results a large set of $\mathrm{TU}_{\mathrm{n}}(0$ to 6561) when compared to LBP ( 0 to 255 ) on a $3 \times 3$ neighborhood. To reduce dimensionality, while preserving the significant information this paper divided the ULBP ternary neighbourhood in to dual neighbourhoods and named them as cross-ULBP (CULBP) and diagonal-ULBP (DULBP). The CULBP and DULBP of a $3 \times 3$ neighbourhood consist of four pixels (Fig. 4). Multiplying each of the four ternary pattern elements of the CULBP and DULBP with ternary weights and concatenating them will result a code that ranges from 0 to $81\left(3^{4}\right)$ as given in Eqn. 7 and 8 . The CULBP and DULBP on a $3 \times 3$ grid can be represented in four ways as shown in Fig.5.1 and Fig.5.2 and this allows the formation of ULBP-DTM in 16 different ways. The ULBP-DTM is formed by placing CULBP code on the $x$ axis and DULBP code on the $y$-axis as in Fig.4. This ULBP-DTM has elements of relative frequencies of both basic windows and has a fixed size of $81 \times 81$. Since the values of $\mathrm{CULBP}_{n}$ and $\mathrm{DULBP}_{\mathrm{n}}$ range from 0 to 80 , the ULBP-DTM will have a fixed size of $81 \times 81$. This reduction is useful to reduce computational complexity of a GLCM. The GLCM method [40] gives reasonable texture information of an image that can be obtained from two pixels. The GLCM method characterizes the spatial relationship between a pixel and a neighboring pixel at a given specific distance and angle. The size of GLCM 
depends on gray level range of the image. The entire process of transforming an ULBP image neighborhood into ULBP-DTM is shown in Fig.4.

$$
\begin{aligned}
& N_{C T U}=\sum_{i=1}^{4} T_{i} \cdot 3^{i-1} \\
& N_{\text {DTU }}=\sum_{i=1}^{4} T_{i} \cdot 3^{i-1}
\end{aligned}
$$

\begin{tabular}{|c|c|c|}
\hline 12 & 5 & 2 \\
\hline 53 & 10 & 8 \\
\hline 14 & 15 & 10 \\
\hline
\end{tabular}

a) $3 \times 3$ neighborhood with ULBP

\begin{tabular}{|l|l|l|}
\hline 2 & 0 & 0 \\
\hline 2 & & 0 \\
\hline 2 & 2 & 1 \\
\hline
\end{tabular}

b) $\quad \mathrm{Ti}=\{2,0,0,0,1,2,2,2\}$

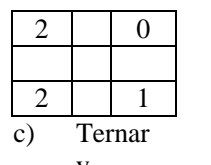

$\mathrm{y}-$

DULB
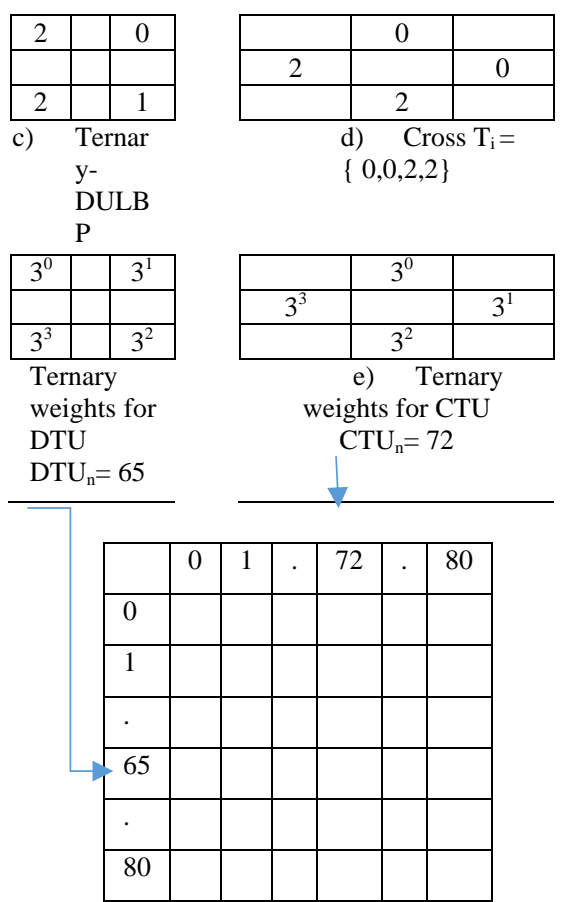

Fig.4. Transformation of ULBP neighborhood into ULBP-DTM (a): ULBP window; b) Ternary representation on ULBP window; c \& d) Ternary elements of DULBP and CULBP window; e \& f) The ternary weights of DULBP and CULBP $\left(\right.$ CULBP $\left._{n} \& \operatorname{DULBP}_{n}\right)$.
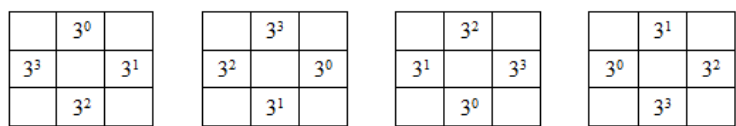

Fig.5.1. Four possible representations of ternary weights of CULBP.

\begin{tabular}{|l|l|l|}
\hline $3^{0}$ & & $3^{1}$ \\
\hline & & \\
\hline $3^{3}$ & & $3^{2}$ \\
\hline
\end{tabular}
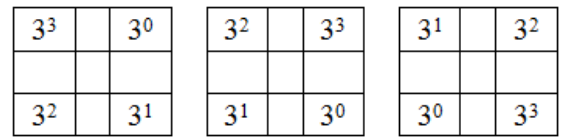

Fig.5.2. Four possible representations of weights of DULBP.

This paper evaluated 13 GLCM features on the ULBPDTM using $0^{\circ}, 45^{\circ}, 90^{\circ}$ and $135^{\circ}$ degrees and performed the classification by computing average of these four rotations. The proposed ULBP-DTM model using GLCM combines the merits of both statistical and structural information of images and thus represents complete information of the facial image.

\section{RESULTS AND DISCUSSIONS}

This paper conducted experiments on three very large scale natural texture databases i.e. Brodtaz [41], UIUC [42] and Outex [43] and the experimental results are compared with other state of art methods CDTM [44], GLCM[40], TS [39], TCTF [7]. The texture images of these databases are natural and captured under varying conditions like illumination, rotation, lightening, reflections etc...The present paper conducted experiments using various machine learning classifiers like Naïvebayes, Multi perceptron, Ibk and J48. This paper listed out the average classification rate, mean absolute error and root mean square error rates.

We selected 24 homogeneous texture image classes from the Brodatz database (Fig. 6). In each class, we considered 10 texture images. The texture images are of size 512 x 512 pixels. We extracted randomly 8 nonoverlapping patches with $128 \times 128$ size from each image. In this way, we generated a dataset containing 1920 (24 x $10 \times 8$ ) texture images, and with each class or category containing 80 images. We have randomly picked 20 texture patch images from each category as training and remaining are used for test images, i.e. $25 \%$ of images are considered for training purpose.
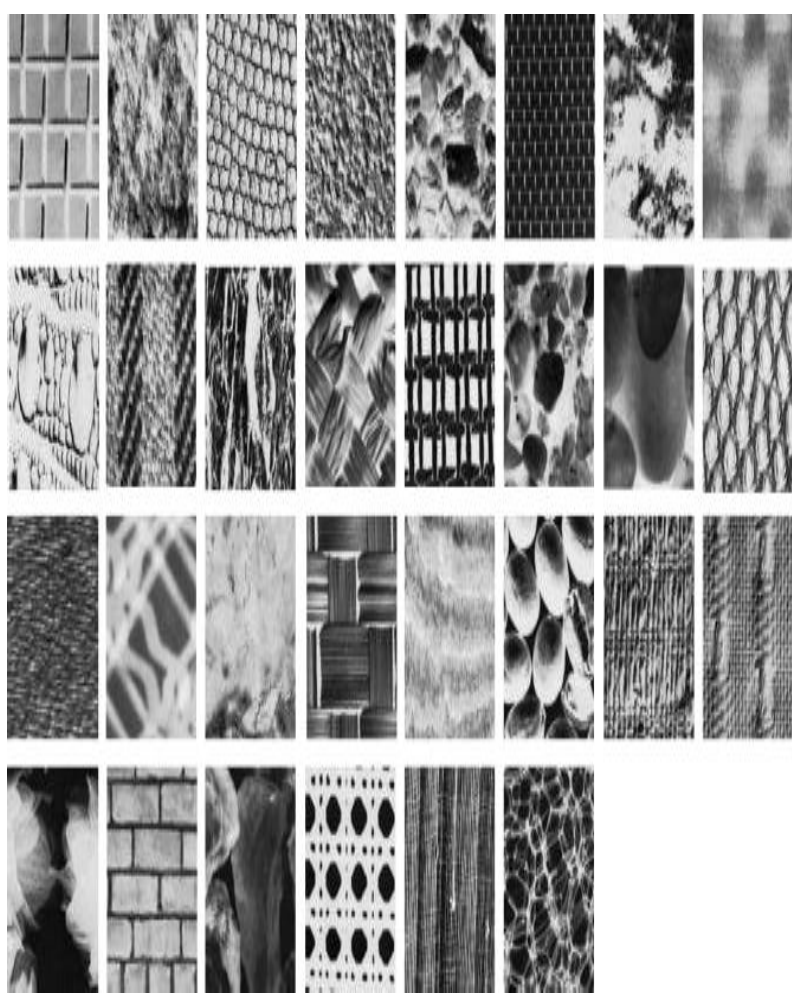

Fig.6. Sample image of Brodtaz textures.

The UIUC texture database is represented with 25 different categories of images, with $640 \times 480$ resolution, shown in Fig.7. The database contains images under significant viewpoint variations. This paper considered 
all 25 classes of UIUC database for experimental sake. And in each category we have randomly picked 10 images. We extracted 10 randomly non overlapped patches from each image with a size of $128 \times 128$. This makes the data base size of 2500 images with resolution of $128 \times 128$ ( $25 \times 10 \times 10)$. In each category we have used 20 texture patches as training set and remaining 80 images as test cases.

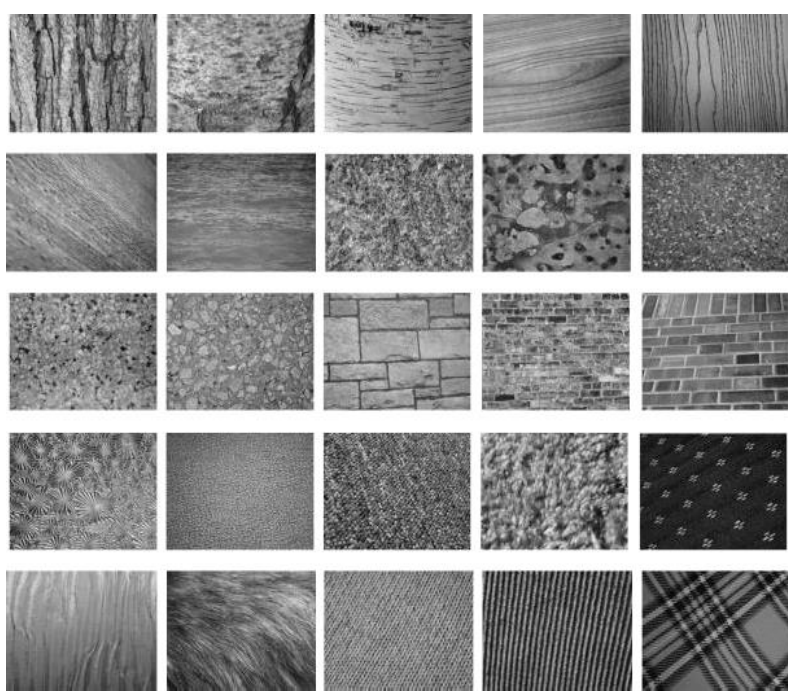

Fig.7. Sample images of the 25 classes from the UIUC database.

The Outex database includes two test suites: Outex_TC_10and Outex_TC_12 (TC12_000 and TC12_001) as shown in Fig. 8. Both TC10 and TC12 contain 24 classes of texture images captured under three illuminations ("inca", "tl84" and"horizon") and nine rotation angles $\left(0^{\circ}, 5^{\circ}, 10^{\circ}, 15^{\circ}, 30^{\circ}, 45^{\circ}, 60^{\circ}, 75^{\circ}\right.$ and $\left.90^{\circ}\right)$. There are twenty $128 \times 128$ images for each rotation angle under a given illumination condition. The $24 \times 20$ images of illumination "inca" and rotation angle $0^{\circ}$ are adopted for the training process. For the TC10 dataset, we use the non-zero rotation angles with illumination "inca" for testing. For TC12 dataset, all the $24 \times 20 \times 9$ samples captured under illumination "tl84" or "horizon" is used as the test data. In order to generate the codebook, we choose one image from each class on TC10 dataset, limiting the training set size to 24 .

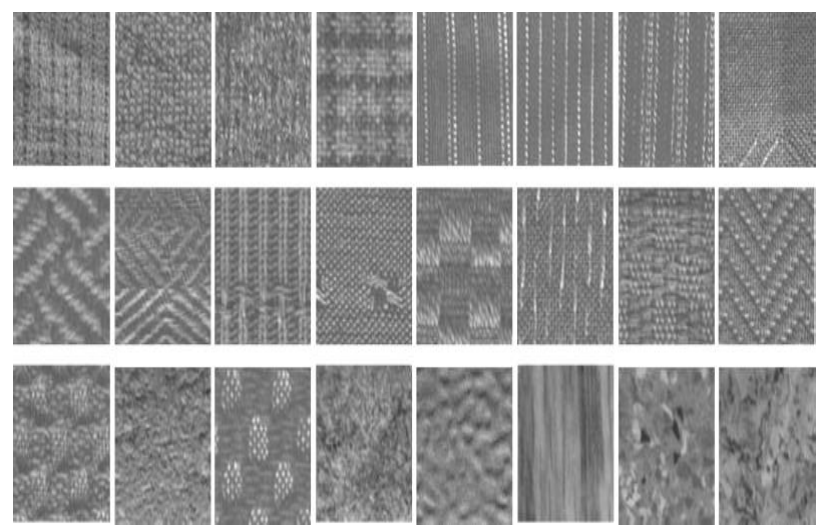

Fig.8. Sample images of the 24 classes from the Outex database.
This paper computed classification rates of textures on ULBP-DTM, using 13 GLCM features on the above three databases using NaiveBayes, Multilayer perceptron, Ibk and J48 classifiers on Weka tools. The 13 GLCM features in four principal directions are given as inputs to the classifiers and the average classification per texture class is listed in Table 1 . We also computed average classification rate of each classifier on ULBP-DTM and presented in the last row of the table. The high classification rate is achieved by multilayer perceptron and in the remaining part of the paper, we will be mentioning this classification rate only on ULBP-DTM.

The paper evaluated the eight TS features [46] on all databases and average classification is listed in the Table 2. The eight TS features are 1) black-white symmetry (BWS), 2) geometric symmetry (GS), 3) degree of direction (DD), 4) micro-horizontal structures (MHS), 5) micro-vertical structures (MVS), 6) micro-first diagonal structures (MDS1), 7) micro-second diagonal structures (MDS2) and 8) central symmetry [46]. This paper also evaluated the 13 GLCM features on the raw texture image using $0^{\circ}, 45^{\circ}, 90^{\circ}$ and $135^{\circ}$ degrees and displayed the classification rate in Table 2 . This paper evaluated the 13 GLCM features on CDTM [44] on 4-different ordering ways and the average classification rate is listed in the Table 2. This paper also computed the texture classification with our earlier method TCBTF [7] approach. The TCBTF [7] approach was only experimented on a particular type or category of textures i.e. stone textures of Brodtaz Texture, however in this paper we have tested the performance of this earlier method [7] on these three types of texture databases with huge number of images.

From Table 2 the following are noted down:

- The eight features derived on texture spectrum has resulted very poor classification rate of $55.36 \%$.

- The TCTF and GLCM features have resulted a classification rate 80.44 and 61.29 respectively.

- The 13 GLCM features on CDTM with average of 4-different orientations has resulted good classification rate of 83.62.

- The proposed ULBP-DTM with the 13 GLCM features outperformed the other existing methods. The proposed ULBP-DTM achieved on average of $90.05 \%$ of classification rate.

- Outex and Brodtaz textures have resulted slightly higher classification rate on all approaches when compared to UIUC database. This is because UIUC database contains lot of orientations and scale changes when compared to other two databases.

The proposed ULBP-DTM exhibited more robustness to monotonic illuminations and other changes in the texture images than other methods due to the following reasons: 
1. The ULBP's are fundamental rotation-invariant properties of local image texture consist of powerful texture feature.

2. The ternary patterns derived on ULBPS resisted to noise and have shown more discriminative power.

3. The division of neighborhoods, into dual neighborhoods reduced dimensionality to a greater extent, and that's why the computational burden of evaluating GLCM features on ULBP-DTM is reduced to a greater extent from $256 \times 256$ to $81 \times$ 81.

4. The GLCM features derived on ULBP-DTM have shown high discriminative power on all databases.
The graphs of Fig. 10, 11 and 12, displays the average classification rate, mean absolute error rate and root mean squared error respectively on four different methods. The proposed ULBP-CDTM is resulted a high classification rate and low error rate on all databases when compared to the other existing method. The proposed ULBP-DTM method achieved on average classification rate of $90.05 \%$, a low average mean absolute error of 0.30 and root mean squared error rate of 0.34 using multilayer perceptron classifier.

Table 1. Classification rate of proposed ULBP- DTM method on different databases using classifiers.

\begin{tabular}{|l|c|c|c|c|}
\hline Databases/Classifiers & NavieBayes & $\begin{array}{l}\text { Multilayer } \\
\text { perceptron }\end{array}$ & Ibk & J48 \\
\hline Brodtaz & 82.13 & 86.48 & 84.78 & 85.69 \\
\hline Outex_T10 & 91.98 & 95.41 & 88.15 & 88.45 \\
\hline Outex_T12 & 90.99 & 94.89 & 87.12 & 85.42 \\
\hline UIUC & 81.78 & 83.43 & 81.15 & 81.78 \\
\hline Avg & 86.72 & 90.05 & 85.30 & 85.33 \\
\hline
\end{tabular}

Table 2. Classification rate of proposed and existing methods.

\begin{tabular}{|l|l|l|l|l|l|l|}
\hline \multicolumn{1}{|c|}{$\begin{array}{l}\text { Databases/ } \\
\text { Classifiers }\end{array}$} & $\begin{array}{l}\text { Proposed } \\
\text { ULBP- } \\
\text { DTM } \\
\text { method }\end{array}$ & CDTM & GLCM & TS & TCTF & $\begin{array}{l}\text { Average } \\
\text { classific } \\
\text { ation } \\
\text { rate per } \\
\text { database }\end{array}$ \\
\hline Brodtaz & 86.48 & 82.46 & 60.18 & 54.76 & 79.56 & 72.68 \\
\hline Outex_T10 & 95.41 & 85.48 & 63.47 & 56.86 & 82.45 & 76.73 \\
\hline Outex_T12 & 94.89 & 87.86 & 64.82 & 57.63 & 84.44 & 77.92 \\
\hline UIUC & 83.43 & 78.69 & 56.71 & 52.21 & 75.32 & 69.27 \\
\hline Avg & 90.05 & 83.62 & 61.29 & 55.36 & 80.44 & \\
\hline
\end{tabular}

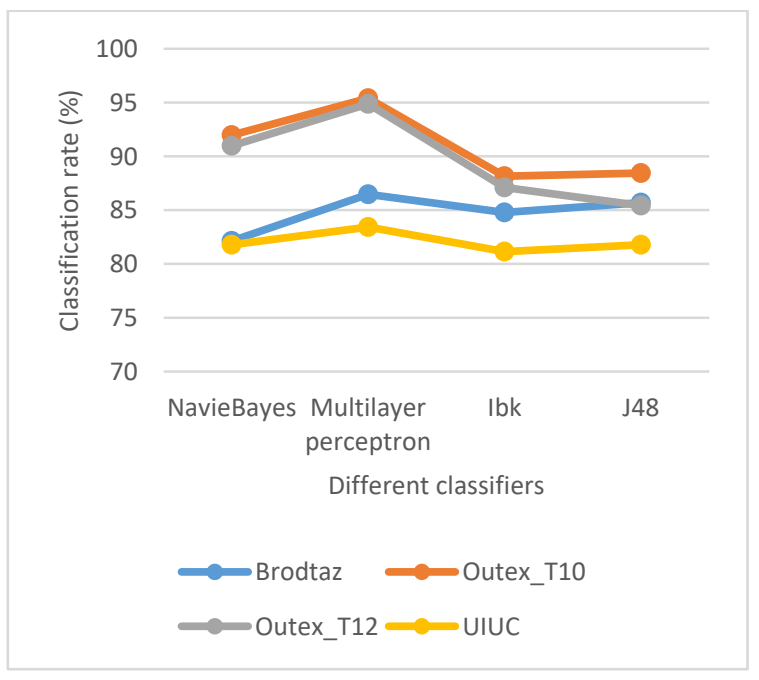

Fig.9. Classification rate of proposed ethod on considered databases.

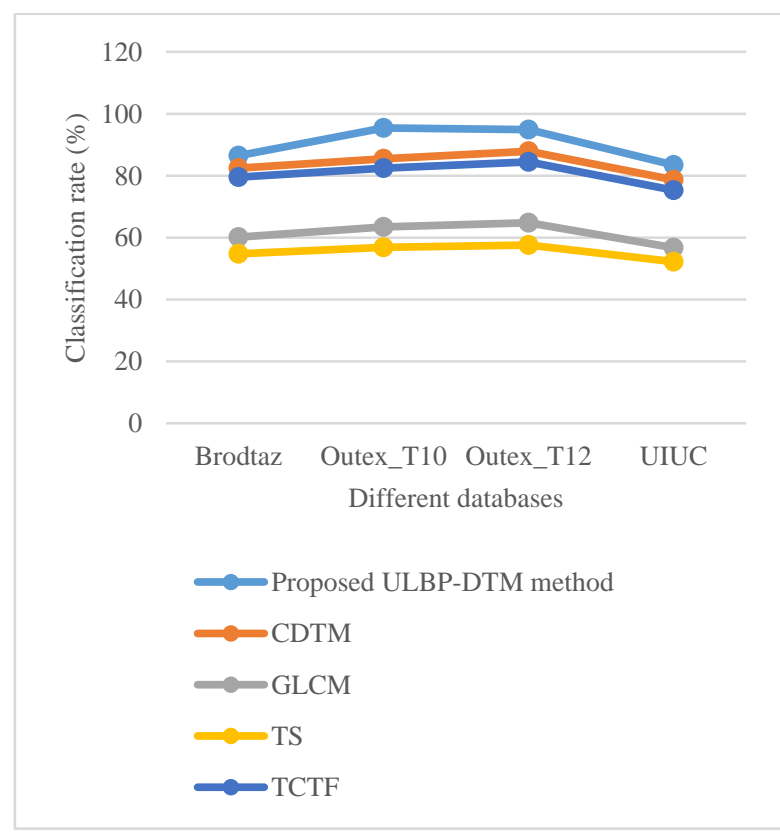

Fig.10. Comparison graph of proposed and existing methods on considered databases in terms of classification rate. 


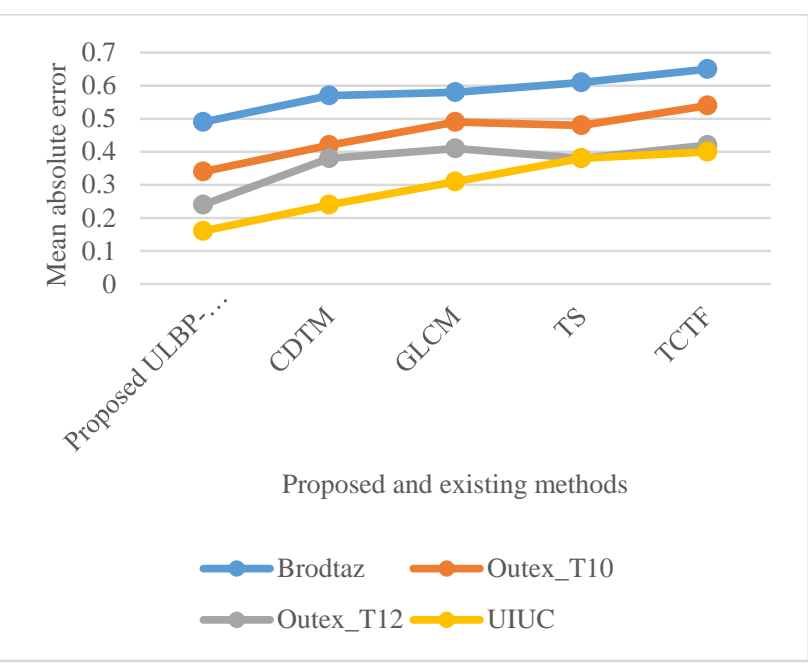

Fig.11. Comparison graph of proposed and existing methods on considered databases in terms of mean absolute error.

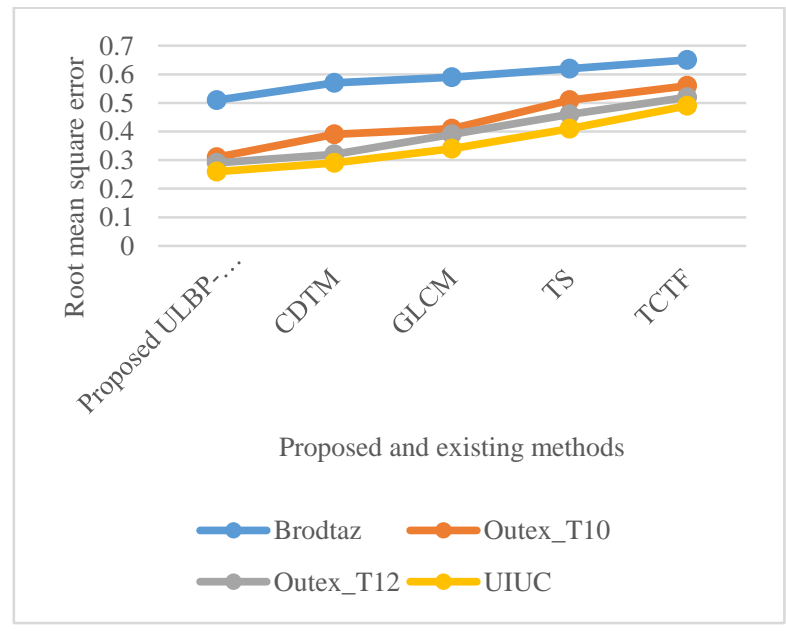

Fig.12. Comparison graph of proposed and existing methods on considered databases in terms of root mean square error.

\section{CONCLUSIONS}

In this paper we integrated the structural and statistical features by deriving a new texture analysis method called ULBP-DTM and it is tested. The present method initially derived ULBP on the textural images to capture rotational invariant fundamental features and treated all 192 NULBP as miscellaneous. By this dimensionality is reduced while preserving the significant and fundamental features of textures. This paper derived ternary patterns on ULBP to reduce the effect of noise and to increase distinctiveness. To reduce the number texture units on ternary patterned texture the present paper divided the $3 \mathrm{x}$ 3 neighborhood in to dual neighborhood and estimated texture units separately and established a relationship among them. This has reduced the dimensionality and derived a texture matrix of size $81 \times 81$ (in case of LBP it is $256 \times 256$ and $6561 \times 6561$ in case of TS). The performance of the proposed method has been compared with GLCM, TS, CDTM and TSTF. The performance of the proposed ULBP-DTM showed better performance and proved to be an excellent gray-scale and rotation invariant texture descriptor.

\section{REFERENCES}

[1] G. Eichmann and T. Kasparis, 1988, "Topologically invariant texture descriptors," Comput. Vis. Graph, Image Process., vol. 41, no. 3, pp. 267-281, Mar.

[2] R. L. Kashyap and A. Khotanzad, 1986, "A model-based method for rotation invariant texture classification," IEEE Trans. Pattern Anal. Mach. Intell., vol. 8, no. 4, pp. 472481

[3] M. Varma and A. Zisserman, 2009, "A statistical approach to material classification using image patch exemplars," IEEE Trans. Pattern Anal. Mach. Intell., vol. 31, no. 11, pp. 2032-2047.

[4] V.Vijaya Kumar, U.S.N. Raju, K. Chandra Sekharan, V.V. Krishna ,2008, "A new method of texture classification using various wavelet transforms based on primitive patterns", ICGST-Graphics, vision and image processing (ICGST-GVIP), Vol.8, No. 2, pp.21-27.

[5] V.Vijaya Kumar, B. Eswar Reddy, U.S.N. Raju, K. Chandra Sekharan, 2007, "An innovative technique of texture classification and comparison based on long linear patterns", Journal of computer science, Science publications, Vol.3, No.8, pp.633-638.

[6] M. Varma, and A. Zisserman, 2005, "A statistical approach to texture classification from single images," International Journal of Computer Vision, vol. 62, no. 1-2, pp. 61-81.

[7] U Ravi Babu, V Vijay Kumar, B Sujatha, 2012, ”Texture classification based on texton features", International journal of image, graphics and signal processing (IJIGSP), Vol.4, No.8, pp.36-42.

[8] B.Eswara Reddy, P.ChandraSekhar Reddy, V.Vijaya Kumar, 2012 "Texton based shape features on local binary pattern for age classification", International journal of image, graphics and signal processing (IJIGSP), Vol. 7, No.4, pp.54-60.

[9] T. Ojala, M. Pietikäinen, and T. Mäenpää, 2002, "Multiresolution gray-scale and rotation invariant texture classification with local binary patterns," IEEE Trans. Pattern Anal. Mach. Intell., vol. 24, no. 7, pp. 971-987.

[10] T. Ojala, M. Pietikäinen, D. Harwood, 1996, “A comparative study of texture measures with classification based on featured distributions”, Pattern Recogn. 29 (1), pp: 51-59.

[11] T. Mäenpää, M. Pietikäinen, 2003, "Multi-scale binary patterns for texture analysis", in: Proceedings of 13th Scandinavian Conference on Image Analysis, G "oteborg, Sweden.

[12] T. Ojala, M. Pietikäinen, T. Mäenpää, 2002, "Multiresolution Gray-Scale and Rotation Invariant Texture Classification with Local Binary patterns", IEEE Trans. Pattern Anal. Mach. Intell. 24 (7), pp: 971-987.

[13] X. Li, W.M. Hu, Z.F. Zhang, H.Z. Wang, 2010, "Heat kernel based local binary pattern for face representation", IEEE Signal Process. Lett. 17 (3), pp: 308-311.

[14] G. Martens, C. Poppe, P. Lambert, R. Van De Walle, 2010, "Noise- and compression-robust biological features for texture classification", Visual Comput. pp: 1-8.

[15] M. Srinivasa Rao , V.Vijaya Kumar, Mhm Krishna PrasadTexture Classification based on First Order Local Ternary Direction Patterns, I.J. Image, Graphics and Signal Processing, 2017, 2, 46-54.

[16] U Ravi Babu, V Vijaya Kumar ,J SasiKiran, 2012, "Texture analysis and classification based on fuzzy 
triangular grey level pattern and run length features", Global journal of computer science and technology graphics \& vision (GJCST), Vol. 12, No. 15, pp. 17-23.

[17] T. Ahonen, A. Hadid, M. Pietikäinen, 2006, "Face description with local binary patterns: application to face recognition", IEEE Trans. Pattern Anal. Mach. Intell.28 (12), pp: 2037-2041.

[18] X. Feng, M. Pietikäinen, A. Hadid, 2005, "Facial expression recognition with local binary patterns and linear programming”, Pattern Recogn. Image Anal. 15 (2), pp: $546-548$.

[19] B.J. Jun, T.W. Kim, D.J. Kim, 2011, “A compact local binary pattern using maximization of mutual information for face analysis", Pattern Recogn. 44 (3), pp: 532-543.

[20] G S Murty ,J SasiKiran , V.Vijaya Kumar, "Facial expression recognition based on features derived from the distinct LBP and GLCM", International Journal of Image, Graphics And Signal Processing (IJIGSP), Vol.2, Iss.1, pp. 68-77,2014, ISSN: 2074-9082.

[21] V.Vijaya Kumar, P.J.S. Kumar, Pullela S V V S R Kumar, 2015, "Age classification of facial images using third order neighbourhood Local Binary Pattern", International Journal of Applied Engineering Research Volume 10, Number 15, pp 35704-35713

[22] G. SatyanarayanaMurth, J.SasiKiran, V. Vijaya Kumar, "Facial expression recognition based on features derived from the distinct LBP and GLCM, I.J. Image, Graphics and Signal Processing (IJIGSP), Vol. 2, 2014, pp: 68-77, ISSN: 2074-9074.

[23] V. VijayaKumar ,Jangala. SasiKiran , V.V. HariChandana, "An effective age classification using topological features based on compressed and reduced grey level model of the facial skin", International journal of image, graphics and signal processing (IJIGSP), , Vol.6, Iss.1, 2013, pp.9-17, ISSN: 2074-9082.

[24] V. Vijaya Kumar, P. Chandra Sekhar Reddy, B. Eswara Reddy, 2015, "New method for classification of age groups based on texture shape features", International journal imaging and robotics, Vol. 15, No.1.

[25] K. Srinivasa Reddy, V.Vijaya Kumar, B.Eshwarareddy, 2015, "Face Recognition based on Texture Features using Local Ternary Patterns", I.J. Image, Graphics and Signal Processing, 10, 37-46

[26] V.Vijaya Kumar, A. Srinivasa Rao, 2015, YK Sundara Krishna, "Dual Transition Uniform LBP Matrix for Efficient Image Retrieval", I.J. Image, Graphics and Signal Processing, 8, 50-57.

[27] X. Tan, and B. Triggs, 2007, "Enhanced Local Texture Feature Sets for Face Recognition Under Difficult Lighting Conditions," in Proc. International Workshop on Analysis and Modeling of Faces and Gestures, pp.168182.

[28] X. Huang, S. Z. Li, and Y. Wang, 2004, "Shape localization based on statistical method using extended local binary pattern," in Proc. International Conference on Image and Graphics, pp.184-187.

[29] S. Liao, M. W. K. Law, and A. C. S. Chung, 2009, "Dominant local binary patterns for texture classification," IEEE Trans. on Image Processing, vol. 18, no. 5, pp. $1107-1118$

[30] M. Heikkilä, M. Pietikäinen, C. Schmid, 2009, "Description of interest regions with local binary patterns," Pattern Recognition, vol. 42, no. 3, pp. 425-436.

[31] K.Srinivasa Reddy, V.V.Krishna, V.Vijaya Kumar, 2015, "Face Recognition using Multi Region Prominent LBP representation", Journal of Information Processing Systems, International Journal of Electrical and Computer
Engineering (IJECE), Vol. 6, No. 6, December 2016, pp. 2781 2788.

[32] X. Qi, R. Xiao, C.-C. Li, Y. Qiao, J. Guo, and X. Tang, 2014, "Pairwise rotation invariant co-occurrence local binary pattern," IEEE Trans. Pattern Anal. Mach. Intell., vol. 36, no. 11, pp. 2199-2213.

[33] S. Hussain and B. Triggs, 2012, "Visual recognition using local quantized patterns," in Proc. Eur. Conf. Comput. Vis., pp. 716-729.

[34] S. H. Lee, J. Y. Choi, Y. M. Ro, and K. N. Plataniotis, 2012, "Local color vector binary patterns from multichannel face images for face recognition," IEEE Trans. Image Process., vol. 21, no. 4, pp. 2347-2353.

[35] Zhibin Pan, Hong cheng Fan, and Li Zhang, 2015, "Texture Classification Using Local Pattern Based on Vector Quantization", IEEE Transactions on Image Processing, Vol. 24, No. 12.

[36] T. Randen and J.H. Husoy, 1990, "Filtering for Texture Classification: A Comparative Study", IEEE Trans. Pattern Analysis and Machine Intelligence, vol. 21, pp. 291-310, 1999.

[37] Ruiz-del-Solar, Rodrigo Verschae, and Mauricio Correa. "Recognition of faces in unconstrained environments: a comparative study." EURASIP Journal on Advances in Signal Processing 2009 (2009).

[38] Samaria, Ferdinando S., and Andy C. Harter. "Parameterisation of a stochastic model for human face identification." In Applications of Computer Vision, 1994. Proceedings of the Second IEEE Workshop on, pp. 138142. IEEE, 1994.

[39] D.C. He, L. Wang, "Texture unit, texture spectrum, and texture analysis", IEEE Trans. Geosci. Remote sens. 28 (4), pp: 509-512.

[40] R. M. Haralick, K. Shanmugam, and I. Dinstein, "Textural features for image classification,” IEEE Trans. Syst., Man, Cybern., Vol. SMC-3, no. 6, pp. 610-621, Nov. 1973.

[41] P. Brodatz, Texture, 1968, "A Photographic Album for Artists and Designers", Reinhold, New York, 1968.

[42] Svetlana Lazebnik, Cordelia Schmid, and Jean Ponce. A Sparse Texture Representation Using Local Affine Regions. IEEE Transactions on Pattern Analysis and Machine Intelligence, vol. 27, no. 8, pp. 1265-1278, August 2005.

[43] http://www.outex.oulu.fi/index.php?page=image_databa se.

[44] Abdulrahman Al-Janobi, "Performance evaluation of cross-diagonal texture matrix method of texture analysis", Pattern Recognition 34, pp: 171-180, 2001.

\section{Authors' Profiles}

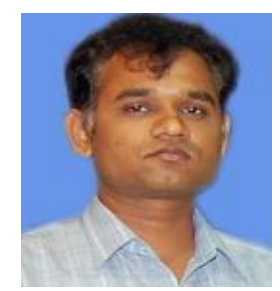

M. Srinivasa Rao received the B.Tech Computer Science \& Engineering from Nagarjuna University in 1998. He completed M.Tech. in Software Engineering from JNT University, MasabTank, and Hyderabad, India in 2001. He is having nearly 15 years of teaching and industrial experience. He is currently working as Associate Professor, Dept of C.S.E, Sri Vasavi Institute of Engineering \& Technology, pedana, Andhrapradesh, India. He is pursuing his Ph.D. from JNT University, Kakinada in Computer Science \& Engineering under the guidance of Dr. V. Vijaya Kumar. He is a life member 
of ISTE and CSI. He published 5 papers in various conferences and journals.

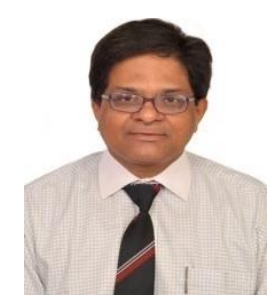

Dr. V. Vijaya Kumar is working as Dean in Dept. of CSE \& IT, chairman Board of studies for CSE and IT and Director Centre for Advanced Computational Research (CACR) at Anurag Group of Institutions, (AGOI) (Autonomous), Hyderabad. He received integrated M.S.Engg, in CSE from USSR in 1989. He received his Ph.D. degree in Computer Science from Jawaharlal Nehru Technological University (JNTU), Hyderabad, India in 1998 and guided 28 research scholars for Ph.D. He acted as principle investigator for various R\&D projects. He has served JNT University for 13 years as Assistant Professor, Associate Professor and Professor. He received best researcher and best teacher award from JNT University, Kakinada, India. His research interests include big data, image analytics, image retrieval, image processing, pattern recognition, digital water marking and cloud computing. At present he is also acting as BoS member for JNTUH and various other institutions. He is the life member of CSI, ISCA, ISTE, IE (I), IETE, ACCS, CRSI, IRS and REDCROSS. He published more than 100 research publications till now in various national, international journals and conferences. He has delivered key note addresses at various international conferences.

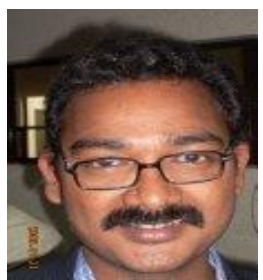

Dr. MHM Krishna Prasad is currently an Professor of the Department of Computer Science and Engineering, University College of Engineering, Kakinada (Autonomous), JNTUK, Andhra Pradesh. He did his B.E. from Osmania University, Hyderabad, M.Tech. and Ph.D. Computer Science and Engineering from JNTU, Hyderabad. He successfully completed a two year MIUR fellowship at University of Udine, Udine, Italy. He has about $50+$ research papers in various International Journals and Conferences, and attended many national and international conferences in India and abroad. He is a member of Association for Computing Machinery (ACM), ISTE and IAENG (Germany) is an active member of the board of reviewers in various International Journals and Conferences. His research interests include data mining, Big Data Analytics and High Performance Computing.

How to cite this paper: M. Srinivasa Rao, V.Vijaya Kumar, MHM KrishnaPrasad,"Texture Classification based on Local Features Using Dual Neighborhood Approach", International Journal of Image, Graphics and Signal Processing(IJIGSP), Vol.9, No.9, pp.59-67, 2017.DOI: 10.5815/ijigsp.2017.09.07 